\title{
Perinatal hormones and genital anesthetization: Effects on masculine sexual behavior of rats
}

\author{
KENNETH W. NIKELS and SHELTON E. HENDRICKS \\ University of Nebraska at Omaha, Omaha, Nebraska 68101
}

\begin{abstract}
Masculine copulatory behavior was evaluated in male and female rats under normal testing conditions and after application of a topical anesthetic to the penis or clitoris. Males had been castrated at birth or at 27 days of age. Females had received either testosterone propionate or control injections during the first 26 days of life. Genital anesthetization prevented the occurrence of intromission and ejaculation patterns in all groups. The masculine sexual behavior observed under conditions of genital anesthetization was comparable across groups, with the exception that females, when tested under these conditions, exhibited lower mount-bout frequencies than did males. However, these differences were not apparent when sexual behavior was evaluated in unanesthetized animals. Females also exhibited longer latencies to mount under anesthetization as compared with control testing conditions. When these differences in latency were taken into consideration, the differences in mount-bout frequency were not apparent. Males that remained intact during development showed shorter latencies to initiate copulatory behavior and more mounts per mount bout as compared with all other groups. The results are interpreted as being consistent with the hypothesis that genital factors account for sex differences observed for androgen-stimulated copulatory behavior in rats.
\end{abstract}

It is well established that the capacity of female rats to exhibit intromission and ejaculatory responses is increased by exposure to testosterone immediately prior to and after birth (Gerall \& Ward, 1966; Ward, 1969; Whalen \& Edwards, 1967; Whalen, Edwards, Luttge, \& Robertson, 1969). It is also clear that neonatal castration of the male rat reduces its capacity to exhibit these responses (Gerall, Hendricks, Johnson, \& Bounds, 1967; Grady, Phoenix, \& Young, 1965; Hendricks, 1969; Whalen \& Edwards, 1967). However, conflicting results are reported for the effects of perinatal hormones when mounting rate is used as an index of masculine behavior (Beach, Noble, \& Orndoff, 1969; Gerall et al., 1967; Gerall \& Ward, 1966; Hendricks, 1969; Nadler, 1969; Sodersten, 1973; Whalen \& Edwards, 1967). Attempts to resolve these inconsistencies are complicated by the fact that mounts, intromissions, and ejaculations are not independent events: Both intromission and ejaculation are followed by periods in which the animal is less likely to mount. Thus, an increasing incidence of intromissions or ejaculations may lower the mount rate, and a decrease in intromissions or ejaculations may raise the mount rate.

Sachs and Barfield (1970) have developed an index of masculine sexual behavior, the mount bout, which appears to be independent of the occurrence of

This research was supported in part by USPHS Grant NS 10027. Steroids were provided by the Schering Corporation. Address requests for reprints to Kenneth Nikels, who is now at the Department of Psychology, Kearney State College, Kearney, Nebraska 68847. intromissions. Mount bouts are defined as "closely spaced attempts at mounting females, separated by periods free of mounting attempts" (Sachs \& Barfield, 1970). Sachs, Pollak, Krieger, and Barfield (1973) found that mount-bout rates are independent of both sex and some perinatal hormone manipulations. The findings that temporal characteristics of masculine sexual behavior (mount or mount-bout frequency) may not be influenced by hormones present during sexual differentiation and that phallic development is affected by such manipulations have led to the suggestion that the effects of perinatal androgenization upon masculine behavior (increased potential for exhibiting intromission and ejaculation) are attributable to differences in genital rather than central nervous system (CNS) development (Beach, 1971; Nadler, 1969; Whalen, 1964; Whalen et al., 1969).

Gray, Davis, and Dewsbury (1976) evaluated the relative contributions of phallic and CNS modifications by testing for masculine behavior under conditions of localized genital anesthesia. Such anesthetization prevented the occurrence of either intromission or ejaculation. Gray et al. (1976) reported that neonatal androgenization of female rats increased mount-bout rates compared with untreated females when sexual behavior tests were conducted under conditions of genital anesthetization. The authors concluded that this finding was "suggestive evidence" that androgen present during the perinatal period acts directly on neural systems that, in adulthood, mediate masculine sexual behavior. However, all groups were not evaluated under both control and 
anesthetization testing conditions. Most significantly, the normal females were tested only under conditions of genital anesthetization. As noted above, Sachs et al. (1973) reported that under normal testing conditions there were no sex differences in mount-bout rates. These findings suggest that genital anesthetization suppresses mount-bout rates in females while having a lesser or no effect in males. The possibility that the anesthetization of the clitoris might have a more profound influence upon the mounting behavior of the female than penile anesthetization would upon the mounting behavior of the male intrigued us. If such were the case, it would raise questions as to the interpretations that can be given to results obtained with a general anesthetization technique. The present study represents a further evaluation of this problem.

\section{METHOD}

\section{Subjects and Treatment Groups}

Subjects were male and female colony-bred rats derived from Long-Evans parent stock (Charles River). Animals were housed under a reversed light-dark cycle (lights on 1700-0700 h). Temperature was controlled between $24^{\circ} \mathrm{C}$ and $28^{\circ} \mathrm{C}$, and food and water were available ad lib. At birth, subjects were sexed and assigned to treatment groups. On the day of birth (Day 1), male subjects were castrated under ice anesthesia or subjected to sham surgical procedures. Females were also anesthetized and subjected to sham surgical procedures. Beginning on Day 2, approximately half of the females received subcutaneous (sc) injections of $200 \mu \mathrm{g}$ of testosterone propionate (TP), dissolved in $.05 \mathrm{ml}$ corn oil, every 3 rd day until they were 26 days of age. The remaining females and the males received the same number of injections of the corn oil vehicle during this period. Animals were weaned at 27 days of age, and the males that had not been castrated at birth were castrated under ether anesthesia. The Day 1 castrated males were subjected to sham surgical procedures. Also at this time, female subjects were ovariectomized under ether anesthesia. The 93 animals that survived to this point included: 23 males castrated at 27 days of age, designated control males (CM); 18 males castrated at Day 1, designated castrated males (CXM); 27 females that received $200 \mu \mathrm{g}$ of TP every 3rd day between Day 2 and Day 26, designated androgenized females (AF); and 25 females that were injected with the oil vehicle between Day 2 and Day 26, designated control females (CF). At 75 days of age, all animals began receiving daily injections of $200 \mu \mathrm{g}$ TP and continued to receive such injections throughout the experiment.

\section{Behavior Tests}

Four weekly masculine-sexual-behavior tests were conducted when the animals were approximately 100 days of age. The four treatment grops were divided so that half of the animals in each group were first tested under conditions of genital anesthetization (Tests 1 and 2) followed by tests under control conditions (Tests 3 and 4). The other half were subjected to the reverse testing sequence. Animals were tested in glass-fronted, semicircular chambers $(65 \mathrm{~cm}$ in diameter). Subjects to be tested were placed in the chambers for an initial 3-min period, after which time genital anesthetization procedures were begun. Genital anesthetization was accomplished by the application of a local anesthetic, Pontocaine (2\% tetracaine hydrochloride, Winthrop), using a cotton swab. The anesthetization procedure was similar to that described by Gray et al. (1976). The phallus was swabbed with Pontocaine for two 60 -sec periods separated by a 60 -sec rest period. Animals in the control condition were swabbed in a like manner, but without Pontocaine. Two minutes after the application procedure, an ovariectomized female, made estrous by the injection of estrogen and progesterone, was placed into the testing chamber. Tests lasted for $15 \mathrm{~min}$, during which time male behaviors were recorded by dictating onto audiotape. Responses were classified following the procedures of Sachs and Barfield (1970). Mounts, with and without intromission patterns, and ejaculation patterns were noted as they occurred. Also, the beginning and end of each mount bout was indicated. Subsequently, the taped record was entered into a computer system preserving temporal relationships.

At 200 days of age, a second series of four male-sexual-behavior tests was conducted. These tests were conducted in the same manner as the first four tests except that all animals were tested after genital anesthetization for the first two tests (Tests 5 and 6) and under control conditions for the last two tests (Tests 7 and 8).

\section{Measures and Analyses}

Measures of male sexual behavior included frequency of mounts (total mounts and mounts with and without intromission patterns), ejaculation, and mount bouts. Furthermore, various temporal characteristics of these behaviors were determined. These latter measures included latencies to mount, intromit, or ejaculate, mountbout duration, inter-mount-bout interval (the time from the start of one mount bout to the start of the next mount bout), timeout (the time from the end of one mount bout to the start of the next), and postejaculatory intervals. Scores obtained from the CM group were statistically compared with scores obtained from each of the other groups. Also, for each group, comparisons were made between scores obtained under control and anesthetization testing conditions. Only animals that exhibited at least two mounts for a given test were considered to have mated, and only data from mating subjects were included in the analyses of a given test. Also, if a rat being tested under anesthetization conditions exhibited an intromission pattern, the data for that animal for that trial were excluded from consideration. This occurred twice and was based upon the premise that the anesthetization procedure had been ineffective. Mann-Whitney U tests were used to compare means in which data took the form of frequency scores, while $t$ tests were employed when mean latencies were compared.

\section{RESULTS}

\section{Behavior Tests 1-4}

Mann-Whitney $U$ tests were used to assess differences in total mount frequencies (i.e., the sum of all mounts exhibited on a particular test whether or not they resulted in intromission patterns). There were no significant differences for this measure between the CM group and any of the other three groups for the first four tests, and there were no significant differences between control and anesthetization conditions. Also, no differences were found in the analyses of the mount-bout data.

The CM group exhibited a higher frequency of intromission patterns on all tests conducted under control conditions when compared with the other three groups (Mann-Whitney U tests, $\mathrm{p}<.01)$. Other than this difference, there were no statistically significant differences for any of the other measures of masculine sexual behavior observed for the first four behavior tests. Because of this lack of reliable differences, data from these tests are given no further consideration. 
Table 1

Mount (M) and Mount Bout (MB) Frequencies Observed During Behavioral Tests Conducted Under Control and Anesthetization Conditions

\begin{tabular}{|c|c|c|c|c|c|c|c|c|c|c|c|c|c|c|c|c|}
\hline \multirow[b]{4}{*}{ Group } & \multicolumn{8}{|c|}{ Anesthetization } & \multicolumn{8}{|c|}{ Control } \\
\hline & \multicolumn{4}{|c|}{ Test 5} & \multicolumn{4}{|c|}{ Test 6} & \multicolumn{4}{|c|}{ Test 7} & \multicolumn{4}{|c|}{ Test 8} \\
\hline & \multicolumn{2}{|c|}{ M } & \multicolumn{2}{|c|}{ MB } & \multicolumn{2}{|c|}{ M } & \multicolumn{2}{|c|}{ MB } & \multicolumn{2}{|c|}{ M } & \multicolumn{2}{|c|}{ MB } & \multicolumn{2}{|c|}{$\mathbf{M}$} & \multicolumn{2}{|c|}{ MB } \\
\hline & Mean & $\mathrm{SE}$ & Mean & $\mathrm{SE}$ & Mean & SE & Mean & SE & Mean & SE & Mean & SE & Mean & SE & Mean & $\mathrm{SE}$ \\
\hline $\mathrm{CM}$ & 24.5 & 4.7 & 12.1 & 1.6 & 27.1 & 4.4 & 12.3 & 1.4 & 11.3 & 2.6 & 10.2 & .9 & 13.0 & 2.8 & 9.5 & .7 \\
\hline CXM & 11.8 & 1.5 & 9.1 & 1.1 & 19.4 & 3.3 & 11.9 & 1.3 & 13.1 & 2.7 & 10.3 & 1.3 & 16.6 & 3.7 & 11.9 & 1.5 \\
\hline $\mathrm{AF}$ & 12.4 & 1.7 & 9.1 & 1.3 & 23.0 & 3.3 & 12.5 & 1.5 & 21.3 & 2.9 & 12.9 & 1.4 & 16.3 & 2.2 & 10.3 & 1.2 \\
\hline $\mathrm{CF}$ & 10.9 & 1.6 & 8.2 & 1.2 & 12.4 & 1.8 & 9.1 & 1.0 & 19.1 & 2.4 & 12.4 & 1.1 & 17.1 & 1.7 & 11.9 & .9 \\
\hline
\end{tabular}

\section{Behavior Tests 5-8}

Mount and mount-bout frequencies from the second set of behavior tests, conducted when animals were 200 days of age, are presented in Table 1. Under conditions of genital anesthetization, the mean number of mounts observed for the CM group was significantly greater $(p<.05)$ than for any other group on Test 5 and significantly greater than that observed for the CF group on Test 6 . There were no significant differences for mount frequency for tests conducted under control conditions. Significant differences in mount frequency between anesthetization and control conditions were found only for the CF group $(p<.05)$, which exhibited higher mount frequencies under control conditions. The CF group also exhibited lower mount-bout rates under anesthetization conditions compared with the CM group $(p<.05)$, and mount-bout frequencies were lower for the $C F$ group under anesthetization as compared with control conditions $(p<.05)$. There were no other significant differences for the mount-bout measure.

A variable that showed large between-groups and between-testing-conditions differences was the latency to the first mount of a test (Figure 1). The latencies of the CM group were significantly shorter than for any of the other groups for tests conducted under both control and anesthetization conditions $(p<.01)$. A comparison of mount latencies observed under anesthetization with those observed under control conditions revealed significant differences only for the CF group, which had significantly shorter latencies under the control conditions $(p<.01)$.

The fact that latency to initiate copulatory behavior varied as a function of both treatment group and conditions of testing suggested that this variable might account for some of the significant differences observed in the frequency data. To assess this possibility, an analysis described by Sachs and Barfield (1970) was employed. For this analysis, only the behavior observed in the $300 \mathrm{sec}$ following the first mount of a test was considered. (The 300 -sec interval was an approximation of the average time for a first ejaculatory sequence in a normal male rat.) The re- sults of this analysis are summarized in Table 2 . In addition to the mean number of mount bouts, two other measures of male sexual behavior are included in this table-the inter-mount-bout interval and timeout. There are no between-groups or between-testingconditions effects for the data presented in Table 2. Similar analyses applied to other measures of masculine sexual behavior, including total mount rate and intermount intervals, also revealed no significant differences for the data obtained during this 300 -sec period.

A measure of copulatory behavior that might be unaffected by differences in mount latency and for which significant between-groups differences were obtained is the number of mounts per mount bout (Figure 2). The CM group exhibited a significantly greater number of mounts per mount bout as compared with the CF group under control and anesthetization conditions. A similar difference was observed for the comparison of the CM with the CXM group, but only for the tests conducted under control conditions $(\mathrm{p}<.05)$.

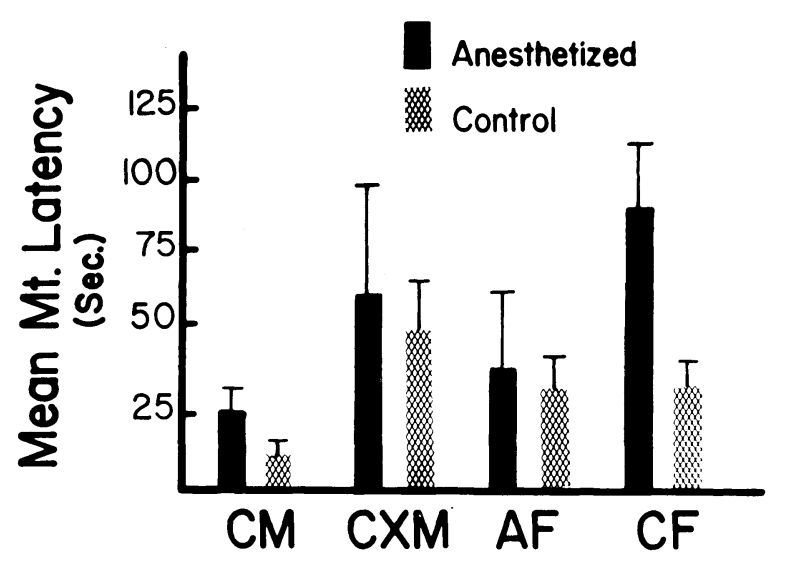

Figure 1. Mean mount latencies observed for the last four masculine-sexual-behavior tests. The means were computed after combining the values from Tests 5 and 6 , which were conducted under anesthetization conditions, and after combining values from Tests 7 and 8, which were conducted under control conditions. 
Table 2

Mean Indexes of Masculine Sexual Behavior Recorded During the $\mathbf{3 0 0} \mathrm{sec}$ Following the First Mount of Tests 5 and 6 (Anesthetized) and Tests 7 and 8 (Control)

\begin{tabular}{|c|c|c|c|c|c|c|c|c|c|c|c|c|}
\hline \multirow[b]{3}{*}{ Group } & \multicolumn{4}{|c|}{ Inter Mount-Bout Interval (in Seconds) } & \multicolumn{4}{|c|}{ Time-Out (in Seconds) } & \multicolumn{4}{|c|}{ Mount Bouts } \\
\hline & \multicolumn{2}{|c|}{ Tests 5 and 6} & \multicolumn{2}{|c|}{ Tests 7 and 8} & \multicolumn{2}{|c|}{ Tests 5 and 6} & \multicolumn{2}{|c|}{ Tests 7 and 8} & \multicolumn{2}{|c|}{ Tests 5 and 6} & \multicolumn{2}{|c|}{ Tests 7 and 8} \\
\hline & Mean & $\mathrm{SE}$ & Mean & SE & Mean & $\mathrm{SE}$ & Mean & SE & Mean & SE & Mean & SE \\
\hline $\mathrm{CM}$ & 57.6 & 5.9 & 68.4 & 3.9 & 40.2 & 6.8 & 33.8 & 4.7 & 6.1 & .6 & 4.7 & .2 \\
\hline CXM & 51.8 & 2.9 & 60.8 & 5.3 & 45.0 & 2.6 & 44.7 & 6.0 & 6.2 & .3 & 5.3 & .4 \\
\hline $\mathrm{AF}$ & 54.3 & 5.1 & 65.1 & 7.7 & 46.1 & 4.8 & 47.1 & 7.7 & 5.5 & .5 & 5.3 & .5 \\
\hline $\mathrm{CF}$ & 63.6 & 4.4 & 56.1 & 3.2 & 58.7 & 4.8 & 44.1 & 2.6 & 5.0 & .5 & 5.9 & .3 \\
\hline
\end{tabular}

\section{DISCUSSION}

Consistent with the results of Gray et al. (1976), we found that intromission and ejaculation responses of rats were largely abolished by applying topical anesthetic to the genitals. Also consistent with Gray et al. (1976), we found that under conditions of genital anesthetization normal females exhibited a lower rate of mount bouts than did normal males. However, the differences between males and females in mountbout rates observed under conditions of genital anesthetization were not apparent when animals were tested under control conditions. This suppression of mount-bout rates by genital anesthetization of females appears to be attributable to differences in the latency to initiate copulatory behavior. When data from only the 300 -sec period following the first mount are considered, the differences in mount-bout rate are not apparent.

We feel that the present data can be interpreted to indicate, as previously found by Sachs et al. (1973),

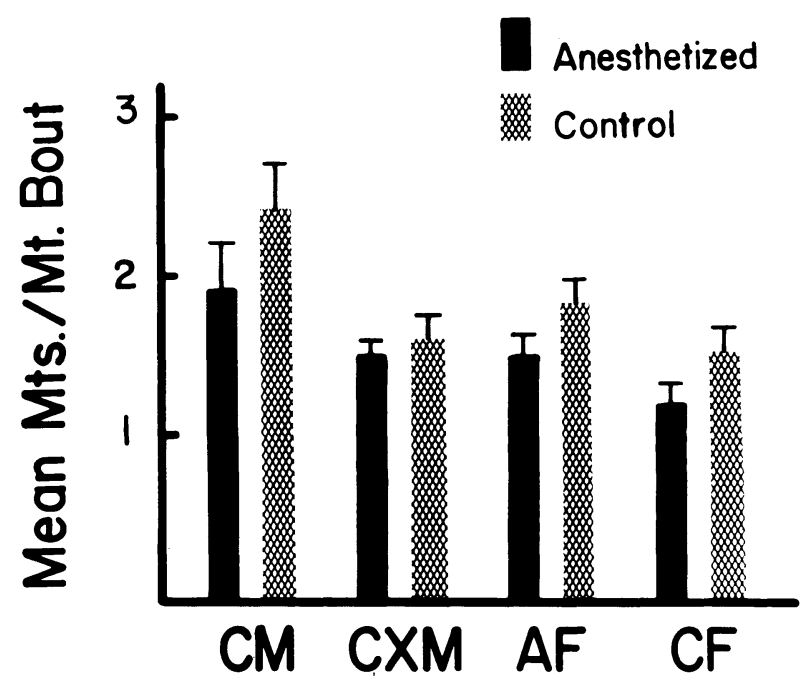

Figure 2. Mean mounts per mount bout observed for the last four masculine-sexual-behavior tests. The means were computed after combining the values from Tests 5 and 6 , which were conducted under anesthetization conditions, and after combining the values from Tests 7 and 8, which were conducted under control conditions. that there are no differences in the frequencies of mount bouts exhibited by male and female rats in response to injected TP. The capacity to exhibit intromission and ejaculation responses, the latency to exhibit copulatory behavior, and the number of mounts per mount bout did vary as a function of gender and/or perinatal hormonal manipulations. A question of interest is: What sex differences in structure or tissue function need be postulated to account for these behavioral differences?

As noted above, the capacity to intromit is greatly influenced by the availability of appropriate peripheral structures. Sex differences in this capacity appear to be attributable primarily, if not solely, to genital differences (Hart, 1972; Nadler, 1969; Whalen, 1964; Whalen et al., 1969). However, in the case of ejaculatory responses, while penile development is an important component, it does not appear to be sufficient to assure normal ejaculatory capacity. Hart (1972) demonstrated that fluoxymesterone, an androgen that maintains penile development without apparent effects on the CNS, injected into neonatally castrated male rats resulted in animals that had normal phallic development and exhibited normal patterns of intromission but were incapable of ejaculating.

The latency to mount is also affected by the state of the genital organs. The present results suggest that clitoral feedback in the female may be more important in this regard than penile feedback in the male. A similar result was observed with respect to the mounts per mount-bout measure. This greater effect of clitoral anesthetization in the female as compared with penile anesthetization in the male might be taken as evidence of some CNS differences. In the absence of genital feedback, other stimuli might become more critical. If, for example, the effects of olfactory stimuli on systems mediating the arousal of masculine sexual behavior were greater in the male, it might explain why interference with genital input would have lesser effects on sexual arousal in the male. Another possible explanation for these differences is provided by the work of Dahlof and Larsson (1978). These investigators demonstrated that the effects of penile deafferentation on male sexual behavior were, in part, a function of the animal's social and copu- 
latory experiences during pre- and early postpuberal development. Specifically, the facilitating effects of previous social experience observed in animals subsequently subjected to penile deafferentation were greater than the previously reported effects of social experience in intact male rats. Thus, there appears to be an interaction between social experience and penile condition, such that social and experiential variables are more important when sexual behavior is evaluated under conditions of impaired genital feedback. Similar findings for copulatory experience are reported by Gray et al. (1976). The social conditions were controlled in the present experiment. However, the CM group was exposed to social interactions and copulatory experiences (Tests 1-4) while possessing normal male genital organs. When confronted with a mating situation under conditions of impaired genital feedback, the CM group may have exhibited less profound deficits because these animals spent their formative days as relatively intact (albeit castrated at 27 days of age) and androgenstimulated (birth to 27 days and 75 days on) males.

In summary, we see marked similarities in the testosterone-stimulated masculine sexual behavior of rats, regardless of sex. In rats, the process of sexual differentiation clearly involves the modification of numerous structural and functional characteristics of the CNS (for a recent review, see Gorski, 1979). Although they do not have correlated effects on the development of genital structure, gonadal hormones, administered neonatally to intact male rats, can suppress the testosterone-stimulated masculine sexual behavior observed in adulthood (Feder, 1967; Harris \& Levine, 1962; Hendricks \& Gerall, 1970; Whalen, 1964; Zadina, Dunlap, \& Gerall, 1979). However, it would appear that these elevations of steroid levels in neonatal males are not reflective of events occurring during normal sexual differentiation (Zadina et al., 1979). With the exception of the ejaculatory response (Hart, 1972), there appear to be few data in the literature that require or strongly suggest that CNS changes be postulated to account for the differences in masculine behavior potential occurring as a consequence of sexual differentiation. We feel that the present data, with respect to the effects of genital anesthetization, support the view that peripheral-specifically, genital-factors are critical in accounting for sex differences in testosterone-stimulated masculine behavior in rats.

\section{REFERENCES}

ВеАсн, F. A. Hormonal factors controlling the differentiation, development and display of copulatory behavior in the ramstergig and related species. In E. Tobach, L. R. Aronson, \& E. Shaw (Eds.), The biopsychology of development. New York: Academic Press, 1971.
Bench, F. A., Noble, R. G., \& Orndoff, R. K. Effects of perinatal androgen treatment on responses of male rats to gonadal hormones in adulthood. Journal of Comparative and Physiological Psychology, 1969, 68, 490-497.

Dahlöf, L., \& Larsson, K. Copulatory performance of penile desensitized male rats as a function of prior social and sexual experience. Behavioral Biology, 1978, 24, 492-497.

Feder, H. H. Specificity of testosterone and estradiol in the differentiating neonatal rat. Anatomical Record, 1967, 157, 79-86.

Gerall, A. A., Hendricks, S. E., Johnson, L. L., \& Bounds, T. W. Effects of early castration in male rats on adult sexual behavior. Journal of Comparative and Physiological Psychology, 1966, 62, 370-375.

Gerall, A. A., \& Ward, I. L. Effects of prenatal exogenous androgen on the sexual behavior of the female albino rat. Journal of Comparative and Physiological Psychology, 1966, 62, 370-375.

Gorski, R. A. Nature of hormone action in the brain. In T. H. Hamilton (Ed.), Ontogeny of receptors and reproductive hormone action. New York: Raven Press, 1979.

Grady, K. L., Phoenix, C. H., \& Young, W. C. Role of the developing rat testis in differentiation of the neural tissues mediating mating behavior. Journal of Comparative and Physiological Psychology, 1965, 59, 370-375.

Gray, G. D., Davis, H. N., \& Dewsbury, D. A. Masculine sexual behavior in male and female rats following perinatal manipulation of androgen: Effects of genital anesthetization and sexual experience. Hormones and Behavior, 1976, 7, 317-329.

Harris, G. W., \& Levine, S. Sexual differentiation of the brain and its experimental control. Journal of Physiology, 1962, 163, 42P-43P.

HART, B. L. Manipulation of neonatal androgen: Effects on sexual responses and penile development in male rats. Physiology \& Behavior, 1972, 8, 841-845.

HENDRICKS, S. E. Influence of neonatally administered hormones and early gonadectomy on rats' sexual behavior. Journal of Comparative and Physiological Psychology, 1969, 69, 408-413.

Hendricks, S. E., \& Gerall, A. A. Effect of neonatally administered estrogen on development of male and female rats. Endocrinology, 1970, 87, 435-439.

NADLER, N. D. Differentiation of the capacity for male sexual behavior in the rat. Hormones and Behavior, 1969, 1, 53-63.

SAChs, B. D., \& BARField, R. J. Temporal patterning of sexual behavior in the male rat. Journal of Comparative and Physiological Psychology, 1970, 73, 359-364.

Sachs, B. D., Pollak, E. I., Krieger, M. S., \& Barfield, R. J. Normal male patterning in androgenized female rats. Science, 1973, 181, 770-772.

Sodersten, P. Increased mounting behavior in the female rat following a single neonatal injection of testosterone propionate. Hormones and Behavior, 1973, 4, 1-17.

WARD, I. L. Differential effect of pre- and postnatal androgen on the sexual behavior of intact and spayed female rats. Hormones and Behavior, 1969, 1, 25-36.

WhALEN, R. E. Hormone-induced changes in the organization of sexual behavior of the male rat. Journal of Comparative and Physiological Psychology, 1964, 57, 175-182.

Whalen, R. E., \& EDwards, D. A. Hormonal determinants of the development of masculine and feminine sexual behavior in male and female rats. Anatomical Record, 1967, 157, 173-180.

Whalen, R. E., Edwards, D. A., Luttge, W. G., \& Robertson, R. T. Early androgen treatment and male sexual behavior in female rats. Physiology \& Behavior, 1969, 4, 33-39.

Zadina, J. E., Dunlap, J. L., \& Gerall, A. A. Modifications induced by neonatal steroids in reproductive organs and behavior of male rats. Journal of Comparative and Physiological Psychology, 1979, 93, 314-322.

(Manuscript received December 20, 1980; revision accepted for publication July 16,1981 .) 